\title{
A national retrospective review of neonatal critical care transfers in dedicated critical care transport services in the private sector
}

\author{
M Venter, BTech Emerg Med Care; E Theron, BA Health Sci, BA Soc Services, BA (Hons) Psychol, MA Research Psychol; \\ W Williams, NDip Emerg Med Care, BTech Emerg Med Care; W Khan, MB ChB, MMed (Emerg Med), PGDip Health Sci Educ; \\ W Stassen, BTech Emerg Med Care, MPhil Emerg Med, PGDip Applied Ethics, PhD
}

Division of Emergency Medicine, Faculty of Health Sciences, University of Cape Town, South Africa

Corresponding author: W Stassen (willem.stassen@uct.ac.za)

Background. South Africa (SA) has a shortage of dedicated neonatal critical care services, which are mostly concentrated in urban areas, thus illustrating the need for neonatal critical care transport (CCT) services. Neonates who are transported by teams without the requisite experience and knowledge in neonatal care are at risk of severe adverse events during transport. This has led to the development of dedicated CCT teams by some emergency medical services. There is a paucity of national literature describing the neonatal population who undergo CCT in dedicated services.

Objectives. To describe a sample of neonates who underwent CCT transfer by dedicated CCT services in the private sector in SA.

Methods. This observational cohort study with a retrospective descriptive design sampled all neonatal transfers completed during 1 year (1 January 2017 - 31 December 2017) from the dedicated CCT of the two largest national emergency medical services in SA. Data were extracted from patient report forms by trained data extractors, and subjected to descriptive analysis.

Results. A total of 444 neonates were transferred between the two services. A total of 760 diagnoses were recorded, yielding an average of $\sim 2$ diagnoses per patient. The most prevalent diagnosis was respiratory distress syndrome $(n=139,31 \%)$, followed by congenital heart defects $(n=123,28 \%)$ and prematurity $(n=81,18 \%)$. Patients had an average of $\sim 4$ attachments, with the most prevalent being patient monitoring $(n=677,152 \%)$. Just under half $(n=182,41 \%)$ of patients required ventilatory support. A total of 422 medications were required during transport, yielding a rate of $\sim 1$ medication or infusion per neonate transported. The most common infusion was maintenance $(n=199,45 \%)$, while almost 1 in 10 neonates required inotropic support $(n=33,7 \%)$.

Conclusions. This study provides insight into the demographics, most prevalent diagnoses, and interfacility transfer monitoring needs of neonates being transported in SA by two private dedicated CCT services. The results of this study should be used to inform future specialised neonatal CCT courses and qualifications, as well as the scopes of practice of providers transporting neonates.

S Afr Med J 2021;111(10):981-984. https://doi.org/10.7196/SAMJ.2021.v111i10.15736

The United Nations sought to reduce the global under-five mortality rate by two-thirds by $2015 .{ }^{[1]}$ While many nations have made considerable strides in realising this Millennial Developmental Goal (no. 4), very poor progress has been made in sub-Saharan Africa, including in South Africa (SA). SA has been identified as one of the countries that had a higher neonatal death rate in 2009 than at baseline in 1990, whereafter it improved. ${ }^{[1]}$ There were 10.7 neonatal deaths for every 1000 live births in 2018. ${ }^{[2]}$ One in every three children who die under the age of five does so during the neonatal period. ${ }^{[3]}$

There exists a shortage of specialised neonatal services in $\mathrm{SA},{ }^{[4]}$ and where they do exist, these centres are often concentrated in urban areas. This is especially true of neonatal intensive care. Owing to this shortage, the transfer of critically ill neonates may occur frequently and often over vast distances. ${ }^{[5,6]}$ In high-income countries, critical care transports (CCTs) of neonates are most often undertaken by specialised physician-based teams with additional training. This is not the case in SA, where emergency medical services (EMS) are responsible for all interfacility transfers (IFTs) regardless of the patient's condition, and it is likely to remain this way owing to a shortage of physicians ${ }^{[7]}$ and specialist neonatologists. Consequently, many critical neonates may be transported by EMS personnel with very limited neonatal care experience and knowledge, as well as limited equipment capabilities. ${ }^{[5,6]}$ Numerous international studies describe higher rates of adverse events and increased morbidity when neonates are transported by non-specialist units. Similarly, studies undertaken in SA have reported increased rates of adverse events. ${ }^{[5,6,8]}$

In response to this risk, some EMS in SA have started the process of developing dedicated paramedic-based CCT services. However, these are in short supply and mostly contained in the private sector. The lack of transport between facilities has been cited as one of the top 10 preventable causes of neonatal deaths in SA. ${ }^{[4]}$ Additionally, there currently exist no specialised or standardised training opportunities for paramedics to undertake these CCTs safely and with confidence. ${ }^{\left[{ }^{[9]}\right.}$ The need for dedicated training on the performance of neonatal CCTs has been described previously. One of the first steps to develop training programmes is to understand the patient population that is intended to be served by the graduate. ${ }^{[10]}$

There is a paucity of national literature describing the neonatal population who undergo CCT in dedicated services. Where studies are available, they are either single centre ${ }^{[6,8]}$ or describe the general neonatal population, ${ }^{[5]}$ which includes a high proportion of neonates who are not critically ill. The aim of this study was therefore to describe a sample of neonates who underwent CCT transfer by dedicated CCT services in the private sector of SA. 


\section{Methodology}

We undertook an observational cohort study with a retrospective descriptive design. All neonatal transfers completed during 1 year (1 January 2017 - 31 December 2017) were extracted from the dedicated CCT services of the two largest national EMS in SA.

\section{Study setting}

In SA, non-physician emergency care personnel undertake neonatal IFT. Although many cadres of pre-hospital providers exist, only advanced life support (ALS) providers may undertake the CCT of a neonate. These providers may either be qualified through vocational (1-year certificate course) or higher education (3-year university diploma or 4-year university Honours degree) training. While this changed in 2020, during the study period, certificate and diplomate pre-hospital providers were able to carry out CCT of a neonate, including mechanical ventilation. After 2020, mechanical ventilation of the neonate is reserved for degreed paramedics only. ${ }^{[1,12]}$

There are very few dedicated CCT services in SA, especially in the public sector. ${ }^{[5]}$ To our knowledge, only the Western Cape and Gauteng Departments of Health operate any. Although both services sampled in this study are from the private EMS, each does serve a certain percentage of government patients, either funded by the patients themselves, or dedicated funding agreements between provincial Departments of Health and the service. Both services have dedicated patient CCT services that operate in various geographic locations within SA, including the Western Cape, Gauteng and KwaZulu-Natal provinces. ${ }^{[5]}$

Services are typically crewed by one ALS and one intermediate life-support provider. Combined, these transfer services perform an estimated 2100 patient transfers per annum.

\section{Sample and sampling}

Neonatal CCTs were identified in two ways, commensurate with the patient report form archiving systems of the EMS. For the first EMS, which utilises electronic patient report forms (PRFs), all cases performed by the aeromedical (helicopter and fixedwing) and ground CCT services were identified and extracted into an Excel (Microsoft, USA) spreadsheet. Data were anonymised upon extraction. Only transfers of neonatal patients undertaken by the dedicated services were included. As gestational age was not consistently recorded, we defined 'neonate' as up to the age of 28 days post-partum, rather than by corrected gestational age. All primary (emergency) neonatal cases, cases undertaken by non-dedicated units and instances in which critical data variables were missing were excluded. Return trips of the same neonate (such as for diagnostic purposes) were also excluded.

For the second EMS, anonymised scanned copies of hand-written PRFs from the dedicated ground CCT services were obtained and screened according to the inclusion and exclusion criteria. After specific training in the research aims, objectives, data variables and contents of the PRFs, the data from eligible cases were extracted according to a dedicated, standard data abstraction form, by a clinical data capturer - a senior paramedic student. Regular meetings between the data capturer and investigators were held to ensure the credibility of the extraction process. This approach is in keeping with the guidance on retrospective chart reviews in emergency care, as outlined by Gilbert and Lowenstein. ${ }^{[13]}$

Data related to demographics, patient contact times, patient diagnosis and attachments and medications were extracted and analysed.

\section{Data analysis}

Regardless of the data source, data were extracted onto a spreadsheet and subjected to descriptive analysis. Categorical data are presented as frequency $(n)$ and proportions (\%), and continuous variables as means. In all instances, more than one diagnosis, attachment or medication is possible for one patient. Additionally, proportions are expressed in terms of the number of patients.

Ethical approval was obtained from the Human Research Ethics Committee of the University of Cape Town (ref. no. 754/2018), and from the private EMS organisations.

\section{Results}

During the period 1 January 2017 - 31 December 2017, a total of 444 neonates were transferred between the two services. The majority of patients were male ( $n=245 ; 55.2 \%$ ), while the mean (standard deviation (SD)) time (mins:secs) spent at the receiving facility to ready the patient for transfer was 51:26 (37:15), and the mean (SD) duration of transport (mins:secs) after departure was 45:48 (49:09).

Table 1 outlines the predominant diagnoses of the transferred neonates. A total of 761 diagnoses were recorded, yielding an average of $\sim 2$ diagnoses per patient. The most prevalent diagnosis was respiratory distress syndrome (RDS; $n=139,31 \%$ ), followed by congenital heart defects (CHDs; $n=123,28 \%)$ and prematurity ( $n=81$, $18 \%)$

Table 2 describes the indwelling devices and attachments. A total of 1892 attachments or indwelling devices were recorded, yielding an average of $\sim 4$ attachments per patient transported. The most prevalent attachment was patient monitoring ( $n=677,152 \%)$, of which pulse oximetry $(n=285,64 \%)$ was the most common modality.

\begin{tabular}{ll} 
Table 1. Diagnoses of neonates transported $(\mathbf{N = 7 6 1})^{*}$ \\
\hline Diagnosis & $\boldsymbol{n}(\%)$ \\
\hline Respiratory distress syndrome & $139(31)$ \\
Congenital heart defects & $123(28)$ \\
Patent ductus arteriosus & $33(7)$ \\
Ventricular septal defect & $18(4)$ \\
CHD, not specified & $14(3)$ \\
Atrial septal defect & $9(2)$ \\
Hypoplastic left heart & $8(2)$ \\
Transposition of the great vessels & $6(1)$ \\
Coarctation of the aorta & $5(1)$ \\
Other, $n<5$ & $30(7)$ \\
Prematurity & $81(18)$ \\
Infection & $63(14)$ \\
Neonatal sepsis & $22(5)$ \\
Pneumonia & $18(4)$ \\
Other, $n<5$ & $23(5)$ \\
Necrotising enterocolitis, including perforation & $41(9)$ \\
Birth asphyxia and HIE & $25(6)$ \\
Low birthweight & $17(4)$ \\
Cardiac complaint, excluding CHD & $17(4)$ \\
Convulsions & $14(3)$ \\
Hydrocephalus & $13(3)$ \\
Meconium aspiration syndrome & $12(3)$ \\
Pulmonary hypertension & $12(3)$ \\
Bowel obstruction or abdominal distention & $11(2)$ \\
Other, $n<10$ & $192(43)$ \\
CHD = congenital heart defect; HIE = hypoxic ischaemic encephalopathy. \\
${ }^{*}$ Individual neonates may have $>1$ diagnosis. \\
& \\
&
\end{tabular}


Table 2. Indwelling devices and attachments of neonates transported $(N=1892)^{*}$

\begin{tabular}{ll}
\hline Description & $\boldsymbol{n}(\%)$ \\
\hline Patient monitoring & $677(152)$ \\
Pulse oximetry & $285(64)$ \\
Electrocardiography & $278(63)$ \\
Capnography & $114(26)$ \\
Vascular access & $389(88)$ \\
Peripheral, venous & $292(66)$ \\
Central, venous & $69(16)$ \\
Arterial & $23(5)$ \\
Other, $<5$ & $5(1)$ \\
Ventilation & $182(41)$ \\
Mode not described & $115(26)$ \\
Non-invasive CPAP & $44(10)$ \\
Bag-valve resuscitator & $11(2)$ \\
Other, $<5$ & $12(3)$ \\
Indwelling attachments & $147(33)$ \\
Gastric tube & $125(28)$ \\
Urinary catheter & $20(5)$ \\
Other, $<5$ & $2(0)$ \\
Incubator & $141(32)$ \\
Medication infusion device & $121(27)$ \\
Supplemental oxygen & $111(25)$ \\
Endotracheal intubation & $110(25)$ \\
Other, $<5$ & $14(3)$ \\
CPAP $=$ continuous positive airway pressure. & \\
×ndividual neonates may have $>1$ device or attachment. & \\
&
\end{tabular}

Table 3. Medications infused or administered during transport $(N=422)^{*}$

\begin{tabular}{ll}
\hline Medication & $\boldsymbol{n}(\%)$ \\
\hline Maintenance & $199(45)$ \\
Neonatalyte & $85(19)$ \\
$5 \%$ dextrose in water & $14(3)$ \\
Other, $n<5$ & $100(23)$ \\
Sedation & $57(13)$ \\
Midazolam & $46(10)$ \\
Ketamine & $8(2)$ \\
Other, $n<5$ & $3(1)$ \\
Inotropes & $33(7)$ \\
Dobutamine & $16(4)$ \\
Dopamine & $8(2)$ \\
Adrenaline & $5(1)$ \\
Other, $n<5$ & $4(1)$ \\
Analgesia & $27(6)$ \\
Antibiotics & $21(5)$ \\
Ampicillin & $7(2)$ \\
Amikacin & $5(1)$ \\
Other, $n<5$ & $9(2)$ \\
Prostaglandins & $18(4)$ \\
Dextrose, $>5 \%$ concentration & $11(2)$ \\
Heparin and saline & $10(2)$ \\
Parenteral nutrition & $8(2)$ \\
Sodium bicarbonate cocktail & $8(2)$ \\
Other, $n<5$ & $30(7)$ \\
${ }^{*}$ Individual neonates may receive $>1$ medication. & \\
&
\end{tabular}

Hereafter, vascular access $(n=398,88 \%)$ in the form of peripheral venous access $(n=292,66 \%)$ was most common. Just under half ( $n=182,41 \%)$ required ventilatory support.

Table 3 describes the medications infused or requiring administration during transport. A total of 422 medications were required during transport, yielding a rate of $\sim 1$ medication or infusion per neonate transported. The most common infusion was maintenance fluid $(n=199 ; 45 \%)$ of which Neonatalyte $(n=85,19 \%)$ was the most prevalent. This was followed by sedation $(n=57,13 \%)$ with Midazolam $(n=46,10 \%)$, while almost 1 in 10 neonates required inotropic support $(n=33,7 \%)$, most commonly with dobutamine $(n=16,4 \%)$.

\section{Discussion}

This study aimed to describe a sample of neonatal patients who underwent IFT by two national dedicated CCT services in the private sector. The five most common diagnoses in this sample were RDS, CHDs, prematurity, infection and necrotising enterocolitis (NEC), while monitoring, vascular access and ventilation were the most common attachments requiring care during transport. Patients had at least one infusion for the transfer, with maintenance fluids, sedation and inotropes the most common. The multiple diagnoses, infusions and attachments highlight the complexity of transporting neonates.

A previous prospective study also reported the most common diagnoses of neonates being transferred in the public sector of KwaZulu-Natal Province to be prematurity, RDS and infection. ${ }^{[5]}$ While not in the neonatal population, these results are echoed in another single-centre, retrospective study from the same province, highlighting respiratory diagnoses to be the most common for children $>1$ month old, admitted to the paedicatric intensive care unit. $^{[6]}$ A 2020 survey similarly demonstrated these diagnoses (including unspecified congenital abnormalities) as the most common diagnostic reasons for transfer of critically ill neonates in the Western Cape Province. ${ }^{[14]}$ It is important to note that prematurity, congenital abnormalities and infection were also among the top causes of neonatal deaths reported at all levels of care of referring facilities nationally, and that lack of IFT resources has been cited as one of the top 10 causes of potentially avoidable neonatal death in SA. ${ }^{[4]}$ This highlights the importance of investment into and development of neonatal transport services.

It is encouraging to note that almost all neonates had continuous monitoring attached, with pulse oximetry being the most common modality. Pulse oximetry in particular plays an important role in avoiding the deleterious effects of hyperoxia, ${ }^{[15,16]}$ such as retinopathy, bronchopulmonary dysplasia and complicating ductal-dependent CHDs. Vascular access and mechanical ventilation were also common attachments. Of note in the public sector, a ventilator and ventilator circuits were not available for $>10 \%$ of transfers. An incubator, also a common attachment in this sample, was not available in almost $20 \%$ of neonatal transfer requests. ${ }^{[5]}$ It is important to keep in mind that our data are from dedicated neonatal transfer services in the private sector, and therefore represent a higher-resourced service when compared with other data.

Since 2020, only degreed paramedics (emergency care practitioners) are licensed to transport neonates who are ventilated. ${ }^{[1,12]}$ With such a high proportion of patients requiring mechanical ventilation, these changes in the scope of practice might have important implications for resource availability in neonatal transport, considering the proportionately low number of qualified emergency care practitioners. ${ }^{[17]}$ Time delays and lack of appropriate skills or equipment have been cited as some of the 
largest challenges to paediatric IFT. ${ }^{[5,17]}$ This further highlights the need for dedicated transport services.

Almost all medications administered during transfer are currently in the scope of practice of pre-hospital providers. In 2017, when the data were collected, these were not included in the scope of practice. ${ }^{[11,12]}$ Some medications, such as prostaglandins, are not expressly covered in undergraduate training, thereby potentially affecting patient safety in transfer. It has been shown that lack of training and experience have been linked to increased adverse event rates during transport. ${ }^{[5,6,8]}$

Improving the knowledge and skills of pre-hospital providers in the CCT of neonates is essential to improve patient safety and outcome. ${ }^{[5,6,8]}$ In a sample of paramedics, just under half reported that their training in neonatal care was either inadequate or extremely inadequate, and reported feeling unprepared to undertake these transfers. ${ }^{[14]}$ Lack of education and training has also been highlighted as an important challenge for pre-hospital providers who undertake these transfers. ${ }^{[17]}$ There is therefore an urgent need for the development of additional qualifications for providers who undertake neonatal CCTs. The exact format of such a qualification, however, is yet to be determined. A previous study ${ }^{[18]}$ has also suggested that an additional scope of practice is required to support the CCT of a neonate, and this suggestion is in line with the patient demographic and attachment and medication requirements of the patients described in this study.

It is interesting that, even though we report data from the private sector, results are similar when compared with the public sector across different SA provinces. ${ }^{[5,6,14]}$ This brings into question a commonly held belief that patient profiles differ significantly between the public and private sectors. Importantly, these findings suggest that educational interventions targeted to these conditions may be applicable across different provinces and sectors.

\section{Limitations}

This study has some important limitations. Firstly, it is retrospective in nature, and reports only on patients transported in 2017. Diagnoses reported herein are based on what was written in the patient report forms, and are therefore not confirmed by the receiving facility. Lastly, this study only describes patients transported by two dedicated private services. While results are similar to those reported in previous public sector studies in non-dedicated neonatal transport services, this limits the external validity of our findings. Future studies should describe the patient case mix of public service transfers.

\section{Conclusion}

This study provides insight into the demographics, most prevalent diagnoses and IFT monitoring needs of neonates being transported in SA by two private dedicated CCT services. The results of this study should be used to inform future specialised neonatal CCT courses and qualifications, as well as the scopes of practice of providers transporting neonates.
Declaration. This study was undertaken in partial fulfilment of the degree of MPhil Emergency Medicine by MV.

Acknowledgements. None.

Author contributions. MV conceptualised the study, collected and analysed data and made critical revisions to the final manuscript. ET analysed data and approved the final manuscript. WW analysed data and made critical revisions to the final manuscript. WK made critical revisions to and approved the final manuscript. WS conceptualised the study, analysed data and drafted, revised and approved the final manuscript.

Funding. The study was self-funded by MV.

Conflicts of interest. None.

1. Damian DJ, Njau B, Lisasi E, Msuya SE, Boulle A. Trends in maternal and neonatal mortality in South Africa: A systematic review. Syst Rev 2019;8(1):76. https://doi.org/10.1186/s13643-0190991-y

2. Masaba BB, Mmusi-Phetoe RM. Neonatal survival in sub-Sahara: A review of Kenya and South Africa. J Multidiscip Healthc 2020;13:709-716. https://doi.org/10.2147/jmdh.s260058

3. Bamford LJ, McKerrow NH, Barron P, Aung Y. Child mortality in South Africa: Fewer deaths, but better data are needed. S Afr Med J 2018;108(3a):S25-S32. https://doi.org/10.7196/SAMJ.2018. v108i3.12779

4. Rhoda N, Velaphi S, Gebhardt G, Kauchali S, Barron P. Reducing neonatal deaths in South Africa: Progress and challenges. S Afr Med J 2018;108(3):S9-S16. https://doi.org/10.7196\%2FSAMJ.2017. v108i3b.12804

5. Ashokcoomar P, Naidoo R. An analysis of inter-healthcare facility transfer of neonates within the eThekwini Health District of KwaZulu-Natal, South Africa. S Afr Med J 2016;106(5):514-518. https://doi.org/10.7196/samj.2016.v106i5.8554

6. Royal C, McKerrow NH. A retrospective review of the transfer of critically ill children to tertiary care in KwaZulu-Natal, South Africa. S Afr J Child Health 2015;9(4):112-118. https://doi. org/10.7196\%2FSAJCH.2015.v9i4.913

7. Eyal N, Cancedda C, Kyamanywa P, Hurst SA. Non-physician clinicians in sub-Saharan Africa and the evolving role of physicians. Int J Heal Pol Manag 2015;5(3):149-153. https://doi. org/10.15171\%2Fijhpm.2015.215

8. Hatherill M, Waggie Z, Reynolds L, Argent A. Transport of critically ill children in a resourcelimited setting. Intensive Care Med 2003;29(9):1547-1554. https://doi.org/10.1007/s00134-003$1888-7$

9. Venter M, Stanton D, Conradie N, Jordaan L, Venter C, Stassen W. The need for setting standards in critical care transfers. S Afr J Crit Care 2017;33(1):32. https://doi.org/10.7196/SAJCC.2017. v33i1.319

10. Bartholomew LK, Parcel GS, Kok G. Intervention mapping: A process for developing theory and evidence-based health education programs. Heal Educ Behav 1998;25(5):545-563. https://doi. org/10.1177/109019819802500502

11. Health Professions Council of South Africa. Clinical Practice Guidelines for Emergency Care Providers. Health Professions Council of South Africa. Clinical Practice Guidelines for Emergency Care Providers.
Pretoria: HPCSA, 2018. https://www.hpcsa.co.za/Uploads/EMB/CLINICAL\%20PRACTICE\%20 GUIDELINES\%20\%20-\%20PROTOCOLS-\%20JULY\%202018.pdf (accessed 4 October 2021).

12. Health Professions Council of South Africa. Health Professions Council of South Africa Professional Board for Emergency Care Capabilities of Emergency Care Providers. Pretoria: HPCSA, 2011. http://www.hpcsa.co.za/downloads/emergency_care/capabilites_june_2011.pdf $\quad$ (accessed 12 February 2018).

13. Gilbert EH, Lowenstein SR, Koziol-McLain J, Barta DC, Steiner J. Chart reviews in emergency medicine research: Where are the methods? Ann Emerg Med 1996;27(3):305-308. https://doi. org/10.1016/s0196-0644(96)70264-0

14. Ismail E, Naidoo R, Prakaschandra DR. Preparedness of Western Cape advanced life support providers to provide clinical stabilisation and intensive care for neonatal interfacility transfers. Australas J Paramed 2020;17:1-6. https://doi.org/10.33151/ajp.17.781

15. Morgan MC, Maina B, Waiyego M, et al. Pulse oximetry values of neonates admitted for care and receiving routine oxygen therapy at a resource-limited hospital in Kenya. J Paediatr Child Health 2018;54(3):260-266. https://doi.org/10.1111/jpc.13742

16. Walker PJB, Bakare AA, Ayede AI,et al. Using intermittent pulse oximetry to guide neonatal oxygen therapy in a low-resource context. Arch Dis Child Fetal Neonatal Ed 2020;105(3):316-321. https:// doi.org/10.1136/archdischild-2019-317630

17. Vincent-Lambert C, Wade G. Challenges relating to the inter-facility transport of high acuity paediatric cases. Afr J Emerg Med 2018;8(1):29-33. https://doi.org/10.1016/j.afjem.2017.12.001

18. Venter M, Stassen W. The capabilities and scope-of-practice requirements of advanced life support practitioners undertaking critical care transfers: A Delphi study. S Afr J Crit Care 2016;32(2):58.
prest https://doi.org/10.7196/SAJCC.2016.v32i2.275

Accepted 17 May 2021. 